been developed with a bespoke section providing near real time analysis of unprocessed COSD data for the NLCA. This portal currently focusses on data completeness, with plans to add process and treatment data in the near future. We report the results of the first 12 months of data collection using the new system (2015), and have compared this to the last year of LUCADA submissions (2014).

Methods The COSD was submitted monthly by English trusts on patients diagnosed with invasive lung cancer throughout 2015. This raw data was used to populate the data completeness tables on the CancerStats portal. An algorithm was developed to allocate a "trust first seen" to each patient record. Our presentation will include data from the final processed cancer registration records that have been validated using all available data sources within the National Cancer Registration Service (NCRAS). Welsh data submitted via their CANISC system will be available for our presentation.

Results 35,000 individual cases of invasive lung cancer were submitted by English Trusts. Data completeness results are shown in Table 1. Our final presentation will be updated with data from the processed cancer registration records that have been validated using all available data sources within the National Cancer Registration Service (NCRAS), as well as results from Welsh trusts.

Conclusion COSD submissions appear to capture more cases of lung cancer than LUCADA. During this transition period, the quality of the data was less good than previous years with significant variation across organisations. However, data completeness for stage and treatment is expected to be better than indicated since final registered cases use data from a variety of other sources. CancerStats offers the opportunity for teams to monitor their data quality and to iteratively improve their internal processes to deliver robust data for future years, in particular patient factors such as performance status which is not available elsewhere.

\begin{tabular}{lll}
$\begin{array}{l}\text { Abstract P104 Table 1 Comparison of LUCADA (2014) } \\
\text { cancer registration data (2015) }\end{array}$ \\
\hline $\begin{array}{lll}\text { National average (Range by Strategic Clinical } \\
\text { Network) }\end{array}$ & $\begin{array}{l}\text { LUCADA } \\
2014\end{array}$ \\
\hline $\begin{array}{l}\text { Number of } \\
\text { diagnoses }\end{array}$ & 33,465 & 27,995 \\
Gender & $100 \%$ & \\
Ethnicity & $86 \%(70-96)$ & $96 \%$ \\
Performance & $69 \%(59-76)$ & $\mathrm{N} / \mathrm{A}$ \\
Status & & $89 \%$ \\
FEV1\% & $24 \%(9-35)$ & \\
Smoking status & $43 \%(27-57)$ & $20 \%$ \\
Basis of Diagnosis & $95 \%(87-100)$ & $\mathrm{N} / \mathrm{A}$ \\
Pre-treatment & $72 \%(54-77)$ & $99 \%$ \\
stage & & $92 \%$ \\
Treatment & $83 \%(66-93)$ & $57 \%$ \\
modality & & \\
\hline
\end{tabular}

\section{P105 LUNG CANCER STAGING - ARE WE GETTING IT RIGHT?}

J Ibrahim, A Mukhopadhyay, V Lostarakos, A Mahmood, S Khan, N Maddekar, S Bikmalla, A Lea, C Satur, Q Abid, S Ghosh, M Haris. Royal Stoke University Hospital, Stoke-on-trent, UK

10.1136/thoraxjn-2016-209333.248
Introduction Despite significant advances in the diagnostic and staging modalities, lung cancer survival remains poor. Accurate staging and stratification of lung cancer is imperative to appropriate management. We reviewed the accuracy of staging in all patients who underwent surgical resection for confirmed or suspected lung cancer.

Methods Retrospective study of consecutive surgical resections over 5 year period between January 2010 and December 2014; patients referred from other hospitals were excluded due to lack of pre-operative staging information. Surgical database and preoperative diagnostic information was reviewed.

Results 298 patients underwent surgical resection, mean age 68 years (range 26-91), male 150 (50\%). All patients had staging CT. 108 (36\%) had EBUS/Bronchoscopy, 9 (3\%) had pleural aspiration, 39 (13\%) had CT guided lung biopsy, 8 (3\%) other tissue sampling* (pelvic lesion, subcutaneous lymph node, previous wedge biopsy \& exploratory thoracotomy). Mean time from staging CT to resection was 47 days. 48 (16\%) had histo-cytological confirmation of lung cancer prior to resection. 248 (83\%) were primary lung, 17 (6\%) metastatic lung tumours from other primaries (breast, colorectal, bladder and renal), 2 (1\%) lymphoma and $31(10 \%)$ benign. Of the 248 patients with lung primary (see Figure 1), pre-operative staging was available in 234; $60 / 234(26 \%)$ were down staged on post-operative staging, 54 (23\%)) upstaged and 120 (51\%) showed concordance.

Conclusion Despite the use of combined pre-operative assessment, staging accuracy was only 51\% and histo-cytological confirmation of lung cancer was only available in a small number of patients. Every effort should be made by the multidisciplinary team to accurately stage lung cancer to guide appropriate therapeutic intervention.

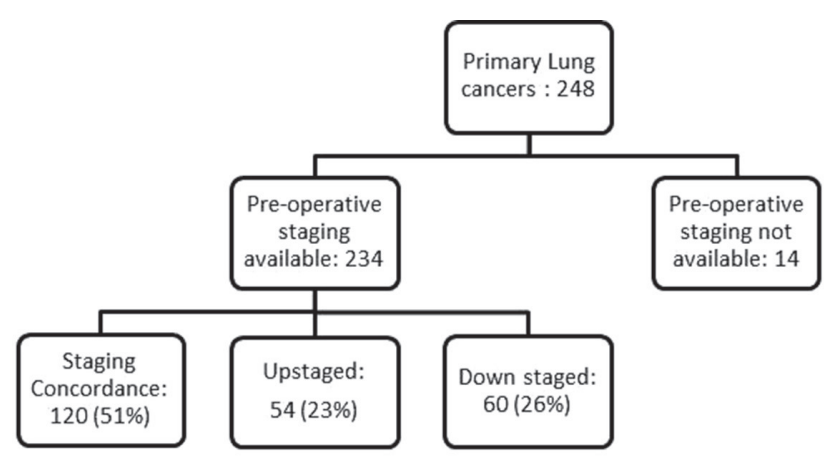

Abstract P105 Figure 1

\section{P106 IMPROVING FIVE YEAR SURVIVAL IN LUNG CANCER: SURVEYING PATIENTS AND CARERS, HCPS AND GPS}

R Steyn, MD Peake. UK Lung Cancer Coalition, London, UK

\subsection{6/thoraxjnl-2016-209333.249}

Introduction The United Kingdom Lung Cancer Coalition (UKLCC) was established in 2005 with the primary goal of doubling five-year survival rates in lung cancer by 2015. Estimates suggest that the UKLCC has met this goal in England, with improvements also seen in Scotland, Wales and Northern Ireland. The UKLCC is now looking to set a new ambition to substantially raise five-year survival rates by 2025 .

Method The UKLCC surveyed 102 patients and carers, 148 healthcare professionals, and 1,003 general practitioners (GPs) asking questions related to improvement of five-year survival rates for lung cancer. 